\title{
Évaluation de la diversité génétique chez les bananiers diploïdes (Musa sp)
}

\author{
F Carreel ${ }^{1}$, S Fauré ${ }^{1}$, D González de León ${ }^{2}$, PJL Lagoda ${ }^{1}$, \\ X Perrier ${ }^{1}$, F Bakry ${ }^{3}$, $\mathrm{H}$ Tezenas du Montcel ${ }^{1}$, \\ C Lanaud ${ }^{1}$, JP Horry ${ }^{1}$ \\ ${ }^{1}$ CIRAD-FLHOR/CIRAD-BIOTROP, \\ BP 5035, F34032 Montpellier Cedex 1, France; \\ 2 CIMMYT Int, Lisboa 27, Colonia Juarez, Apdo Postal 6-641, \\ 06600 Mexico city, DF, Mexique; \\ ${ }^{3}$ CIRAD-FLHOR, Sainte-Marie, F97130 Capesterre Belle-Eau, Guadeloupe
}

\begin{abstract}
Résumé - La majorité des bananiers cultivés sont triplö̈des. Importants en sélection, les bananiers diploïdes sont analysés pour la compréhension de l'organisation génétique du complexe d'espèces Musa. À l'aide de 30 sondes uniques nucléaires cartographiées, le polymorphisme de longueur des fragments de restriction (RFLP) est utilisé pour analyser la diversité génétique de 160 variétés de bananiers diplö̈des : 70 clones séminifêres (sauvages) appartenant aux espèces Musa acuminata, $M$ balbisiana et $M$ schizocarpa et 90 variétés parthénocarpiques (cultivars). Des allèles spécifiques à chacune des 3 espèces ont été trouvés. $M$ acuminata est l'espèce la plus polymorphe; sa structuration est discutée. Il est montré que $M$ schizocarpa est relativement proche de $M$ acuminata banksii, $M$ balbisiana se révélant beaucoup plus éloignée. Tous les diploïdes parthénocarpiques présentent des allèles $M$ acuminata, confirmant le rôle de cette espèce dans l'origine de la parthénocarpie. L'existence de cultivars hybrides $M$ acuminata par $M$ schizocarpa est démontrée.
\end{abstract}

Musa sp / complexe d'espèces / RFLP / taxonomie

Summary - Evaluation of the genetic diversity in diploid bananas (Musa spp). Most cultivated bananas are triploids. Diploid bananas are important to breeding programmes. We analyzed diploids to investigate the genetic organisation of the Musa species complex. Restriction fragment length polymorphism (RFLP) was used to analyze the genetic diversity of 160 diploid bananas: 70 seminiferous clones (wild bananas) of the species Musa acuminata, $\mathrm{M}$ balbisiana and $\mathrm{M}$ schizocarpa and 90 parthenocarpic clones (cultivars) were studied using 30 mapped single copy nuclear probes. Alleles specific to the 3 species studied were found. $\mathrm{M}$ acuminata is the most polymorphic: its genetic structuration is discussed. $\mathrm{M}$ schizocarpa appeared to be very similar to $\mathrm{M}$ acuminata banksii, and $\mathrm{M}$ balbisiana is more divergent. All the diploid parthenocarpic bananas contain $\mathrm{M}$ acuminata alleles. This 
confirms the involvement of $\mathrm{M}$ acuminata in the origin of parthenocarpy. Some cultivars were proved to be hybrids between $\mathrm{M}$ acuminata and $\mathrm{M}$ schizocarpa.

Musa sp / species complex / RFLP / taxonomy

\section{INTRODUCTION}

D'une grande importance alimentaire dans de nombreux pays de la zone intertropicale, les bananiers sont originaires d'Asie du Sud-Est. Le «complexe bananier» (genre Musa) défini par Dodds (1943) regroupe des bananiers de type séminifère (sauvages) et de type parthénocarpique (cultivars).

Les bananiers séminifères ou à graines dits sauvages sont diploïdes $(2 n=22)$; ils se multiplient végétativement et par voie sexuée. Sur les 15 espèces décrites dans la section Eumusa, $M$ acuminata Colla et $M$ balbisiana Colla ont été suspectées très tôt comme étant à l'origine de la plupart des cultivars (Cheesman, 1948). $M$ acuminata présente des types bien différenciés associés à des zones géographiques plus ou moins isolées; une structuration en sous-espèces est globalement admise (Shepherd, 1990). Des observations cytologiques ont montré que les différentes sous-espèces se différencient par la fixation à l'état homozygote d'anomalies structurales chromosomiques de type translocation. Elles ont vraisemblablement limité l'introgression entre sous-espèces, en réduisant la fertilité des hybrides inter-subspécifiques. La diversité morphologique observée chez $M$ balbisiana est moindre que chez $M$ acuminata. Les génomes des 2 espèces ne sont que partiellement homologues (Dessauw, 1987) et les hybrides fortement stériles (Simmonds, 1962).

Les bananiers parthénocarpiques, ou cultivars, sont diploïdes, triploïdes ou plus rarement tétraplö̈des. Ils sont fortement stériles, voire totalement pour certains clones. Ils sont facilement multipliés végétativement. La structuration des cultivars est basée sur l'observation de 15 descripteurs morphologiques différenciant les 2 espèces $M$ acuminata et $M$ balbisiana (Simmonds et Shepherd, 1955). Les cultivars ayant un même niveau de plö̈die et une même contribution relative des 2 espèces à leur expression phénotypique sont regroupés. Chaque groupe est désigné par une formule génomique de 2, 3 ou 4 lettres avec A pour acuminata et B pour balbisiana; les principaux sont les AA, AAA (dont les bananes desserts), AAB (dont les bananes plantains) et ABB.

L'évolution des espèces sauvages vers les bananiers cultivés a pu se faire par l'accumulation de facteurs de stérilité et l'acquisition de la parthénocarpie. L'homme a progressivement sélectionné et propagé par rejets les plantes les plus stériles et parthénocarpiques. L'apparition de la parthénocarpie a toujours été associée à $M$ acuminata. Cependant, les avis divergent quant à l'existence de cultivars parthénocarpiques de constitution purement balbisiana (BB, BBB). Plusieurs facteurs interviendraient dans la stérilité des cultivars. La fertilité de nombreux cultivars diploïdes 'AA' est réduite par la présence à l'état hétérozygote des anomalies structurales des différentes sous-espèces de $M$ acuminata (Fauré et al, 1993a). Une stérilité femelle associée à la parthénocarpie doit y être ajoutée (Dodds, 1943). De plus, la triploïdie de certains cultivars est en elle-même génératrice de stérilité. On 
retiendra également une stérilité due à la non-homologie des génomes acuminata et balbisiana chez les cultivars hybrides.

Une troisième espèce, Musa schizocarpa Simmonds pourrait également avoir joué un rôle dans l'évolution de certains cultivars (Shepherd et Ferreira, 1982). Outre des hybrides interspécifiques séminifères (Argent, 1976), des bananiers parthénocarpiques présentant des caractéristiques de $M$ acuminata et $M$ schizocarpa, ont été observés en Papouasie Nouvelle-Guinée où se côtoient les 2 espèces.

Seuls quelques clones triploïdes sont utilisés dans les bananeraies pour l'exportation. Un plus grand nombre de clones principalement triploïdes mais aussi diploïdes sont cultivés pour la consommation locale soit $88 \%$ de la production mondiale. Toutes ces productions sont particulièrement fragiles face aux menaces parasitaires croissantes.

Le CIRAD-FLHOR a développé un programme d'amélioration génétique des bananiers visant la création de cultivars triplö̈des synthétiques à partir de variétés diploïdes (Bakry et al, 1990). Pour mieux cibler les parents diploïdes séminifêres ou parthénocarpiques intéressants, une meilleure connaissance de la diversité génétique et de la structuration du complexe d'espèces est nécessaire. Les études réalisées à l'aide de marqueurs morphologiques (Simmonds et Weatherup, 1988), polyphénoliques et enzymatiques (Horry, 1989; Lebot et al, 1993) ont montré l'intérêt d'une analyse de la structuration des diploïdes séminifères et parthénocarpiques pour la compréhension de l'organisation génétique des cultivars triploïdes.

Parallèlement à l'étude de la fertilité et de l'aptitude à la combinaison des clones diploïdes, leur diversité génétique a été évaluée à l'aide de marqueurs moléculaires de type RFLP (restriction fragment length polymorphism). Une première étude portant sur le polymorphisme de longueur de l'espaceur intergénique de l'ADN ribosomique (Lanaud et al, 1992) n'a pas montré de structuration basée sur la classification en groupes génomiques. Mais montrant une relation avec l'origine géographique de certains clones, la variation de longueur de l'espaceur serait le reflet de mutations locales plus récentes.

La présente étude a été conduite dans le but de mieux connaître la structuration des bananiers séminifêres et des diploïdes acuminata parthénocarpiques, eux-mêmes à la base de l'évolution des triploïdes. Cent-soixante diploïdes ont été analysés à l'aide de marqueurs RFLP pour mieux comprendre leur organisation génétique.

\section{MATÉRIEL ET MÉTHODES}

Matériel végétal : 160 bananiers diplö̈des : 70 bananiers séminifêres représentant les clones, disponibles en collection, des 3 espèces $M$ acuminata, $M$ balbisiana et $M$ schizocarpa et 90 bananiers parthénocarpiques, ont été analysés (tableau I).

Techniques RFLP : les extractions d'ADN total ont été réalisées selon le protocole d'Hoisington (1992) à l'aide du tampon d'extraction de Gawel et Jarret (1991b). L'ADN a été digéré avec l'une des enzymes de restriction DraI, EcoRV ou HindIII. Les protocoles de restriction, transfert, marquage et hybridation sont ceux de Fauré et al (1993b).

Sondes : sur l'ensemble des sondes cartographiées issues de 2 banques nucléaires partielles de l'espèce Musa (Fauré et al, 1993b), 30 sondes uniques représentant au 
Tableau I. Répartition des clones analysés par RFLP dans les différentes espèces et sousespèces pour les bananiers séminiferes et dans les différents groupes génomiques pour les bananiers parthénocarpiques. Genre : Musa, section : Eumusa.

\begin{tabular}{lrlr}
\hline Séminifères & & \multicolumn{2}{c}{ Parthénocarpiques } \\
\hline schizocarpa & $5(5)$ & & $2(2)$ \\
schizocarpa $\times$ acuminata & $3(3)$ & AS & $84(28)$ \\
acuminata & & AA & \\
$\quad$ banksii & $14(10)$ & & \\
burmannica & $4(0)$ & \\
burmannicoïdes & $1(0)$ & & \\
malaccensis & $1(0)$ & & \\
microcarpa & $4(0)$ & & \\
siamea & $2(0)$ & & \\
truncata & $2(0)$ & & \\
zebrina & $1(0)$ & & \\
$\quad$ indéterminé & $4(0)$ & AB \\
acuminata $\times$ balbisiana & $15(0)$ & BB? \\
balbisiana & $3(2)$ & $8(0)$ & \\
\hline
\end{tabular}

Dans chaque groupe, sur l'ensemble des bananiers analysés, ceux provenant d'une récente prospection organisée par l'INIBAP (International Network for the Improvement of Banana and Plantain) et l'IBPGR en Papouasie Nouvelle-Guinée (Sharrock, 1990) sont indiqués entre parenthèses. Ils proviennent du QDPI, South Johnstone, Qld en Australie. Tous les autres clones proviennent du CIRAD-FLHOR, Capesterre Belle-Eau en Guadeloupe.

mieux les différents groupes de liaison du génome ont été choisies (fig 1). Après un tri préalable sur 4 enzymes, chaque sonde a été associée à l'enzyme montrant les profils polymorphes les plus lisibles.

\section{RÉSULTATS}

L'analyse de 30 couples enzyme/sonde a permis de révéler 212 niveaux de bandes dont 197 sont présents chez au moins 3 clones. Les 15 autres niveaux sont spécifiques de clones, ils n'ont pas été utilisés dans les analyses. Les bananiers sauvages et les cultivars ont pour chaque sonde un nombre comparable de bandes.

\section{Les bananiers séminifêres}

Chaque sonde révèle en moyenne 6 à 7 niveaux de bandes. Une seule sonde (pMaCIR36) ne révèle que 2 bandes dont une se montre spécifique du génome balbisiana. Chez 13 autres sondes, 18 niveaux de bandes sont également absents des génomes acuminata et schizocarpa. Un seul niveau de bande issu de la sonde pMaCIR1024 s'est montré totalement spécifique du génome schizocarpa. Six niveaux de bandes absents chez tous les $M$ balbisiana sont présents chez tous les schizocarpa et chez quelques individus acuminata. 


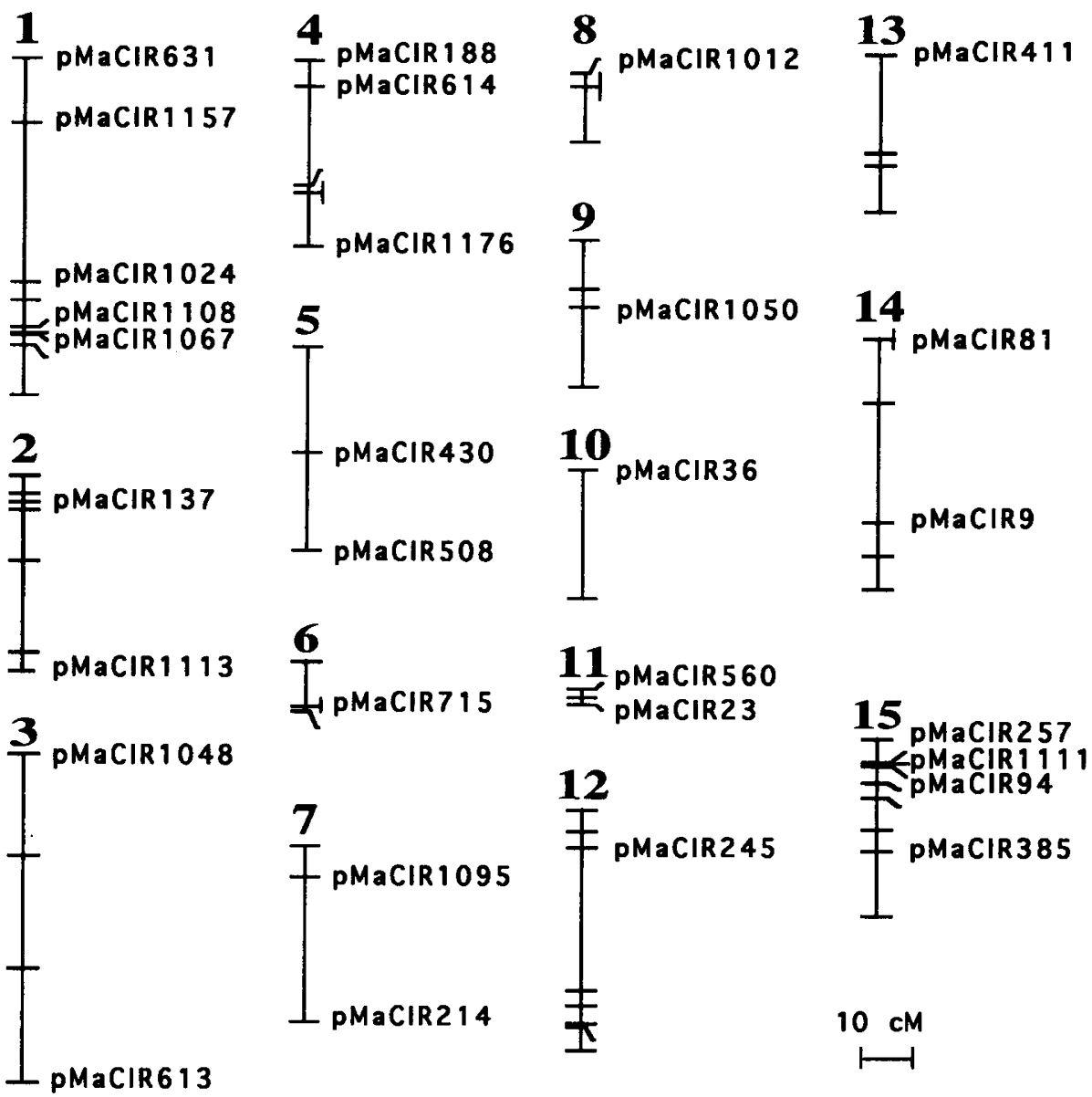

Fig 1. Nom et localisation des 30 sondes utilisées, sur les différents groupes de liaison issus de la cartographie du croisement de NBB11/SF265 $\times M$ a banksii type banksii (Fauré et al, 1993b).

Les niveaux de bandes de 25 des 30 sondes cartographiées utilisées ont pu être interprétés en allèles après vérification sur plusieurs croisements (données non montrées). Les taux d'hétérozygotie moyens des clones des espèces $M$ balbisiana et $M$ schizocarpa sur ces 25 locus sont respectivement de $2 \%$ et de $7 \%$. L'hétérozygotie moyenne chez $M$ acuminata est plus élevée et variable. Seuls les représentants de la sous-espèce $M a$ banksii et l'unique individu de la sous-espèce $M$ a errans se sont révélés fortement homozygotes (taux moyen d'hétérozygotie de 1,8\%). Chez les formes morphologiquement identifiées comme appartenant aux 7 autres sous-espèces, l'hétérozygotie moyenne est de $30 \%$. Les clones de $M$ acuminata pour lesquels on ne peut morphologiquement définir l'appartenance à une sousespèce particulière (formes indéterminées) sont très hétérozygotes (taux moyen 
d'hétérozygotie de 50\%). Les hybrides séminifères interspécifiques : AB et AS sont également très hétérozygotes (taux moyen de $60 \%$ ).

Une analyse factorielle des correspondances (AFC) de 70 bananiers séminifères a été réalisée à partir des 197 niveaux de bandes variables. Les 2 premiers axes (fig 2) expliquent $27 \%$ de la variabilité. Les clones de $M$ balbisiana apparaissent très distincts de ceux des 2 autres espèces. Les clones de $M$ schizocarpa ne se différencient des clones de $M$ acuminata que sur le quatrième axe qui représente $7 \%$ de la variabilité. Sur les 8 clones de l'espèce $M$ balbisiana, 4 clones ont révélé le même profil. Les 5 clones de l'espèce $M$ schizocarpa ont pu être différenciés.

\section{Classification des bananiers parthénocarpiques}

L'existence d'allèles spécifiques aux différentes espèces permet la reconnaissance du groupe génomique auquel appartient une accession. Des erreurs d'affectation ont ainsi pu être décelées. Le cultivar AA-Luba (PNG 146) comporte 9 des 19 niveaux de bandes spécifiques du génome balbisiana. Treize des 19 niveaux de bandes balbisiana sont bien révélés chez les 2 cultivars $\mathrm{BB}$ trouvés identiques dans notre analyse : Aoko (PNG 034) et Auko (PNG 125). Mais ceux-ci comportent de nombreux niveaux de bandes spécifiques à $M$ acuminata et leur niveau d'hétérozygotie est comparable à celui des bananiers séminiferres $\mathrm{AB}$. Ces 3 accessions sont donc à rattacher au groupe AB. Les 2 clones parthénocarpiques AA-Wompa (PNG 063) et AA-Japaraka (PNG 065) comportent l'allèle spécifique schizocarpa et plusieurs allèles principalement observés chez cette espèce. Nous les classons comme cultivars hybrides acuminataschizocarpa ou AS.

\section{Les clones séminifêres et parthénocarpiques acuminata}

Une analyse factorielle des correspondances (AFC) a été réalisée à partir des 197 niveaux de bandes variables sur tous les clones acuminata. Le nombre d'accessions de la sous-espèce $M a$ banksii étant très nettement supérieur à celui des autres sous-espèces, seuls 5 individus ont été utilisés pour la définition des axes factoriels pour ne pas déséquilibrer l'analyse; les 9 individus restants ont été projetés comme individus supplémentaires. Les deux premiers axes de l'AFC expliquent $30 \%$ de la variabilité (fig 3 ).

L'axe 1 de l'AFC oppose les individus $M$ a banksii aux autres sous-espèces de $M$ acuminata. $M$ a errans est rattaché à ce groupe banksii. L'axe 2 différencie les autres sous-espèces de $M$ acuminata en 3 groupes : l'un comprend les sous-espèces $M$ a burmannica et burmannicoides, un deuxième les principaux représentants de $M$ a malaccensis et celui de $M$ a truncata et le troisième comprenant les individus représentatifs des sous-espèces $M$ a zebrina et $M$ a microcarpa. Les représentants de la sous-espèce $M$ a siamea sont répartis entre le premier et le deuxième groupe. La majorité des cultivars issus des prospections en PNG se situe au voisinage des $M$ a banksii. Le taux d'hétérozygotie moyen chez ces cultivars est cependant élevé $(45 \%)$. Aucune structuration forte n'apparait clairement au sein des autres cultivars AA, mais ils présentent un très fort taux moyen d'hétérozygotie $(60 \%)$. 


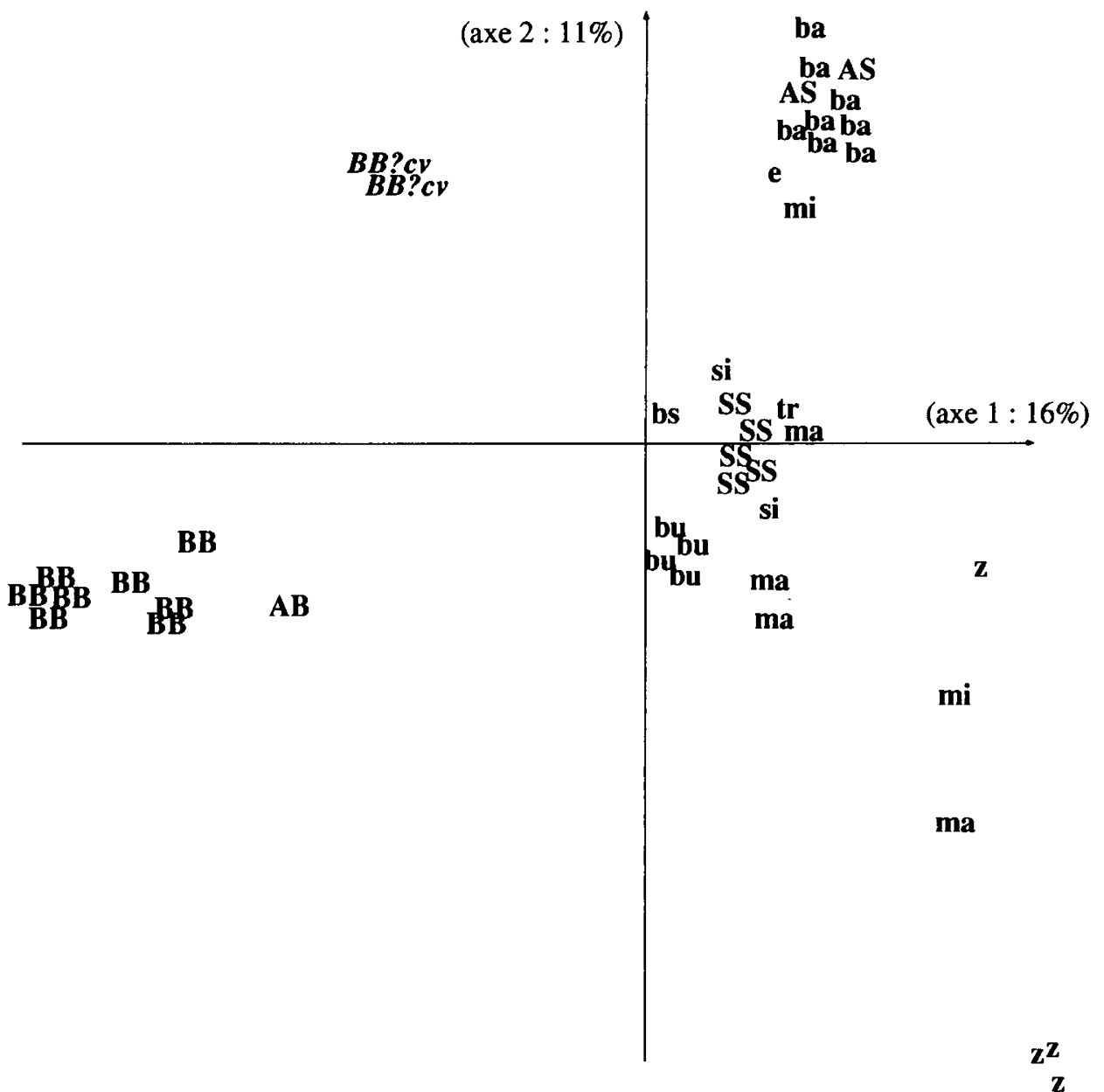

Fig 2. Localisation de 55 bananiers séminiferes sur le premier plan de l'AFC qui représente $27 \%$ de la variabilité révélée par 30 sondes nucléaires. Sont représentés par une majuscule : $M$ balbisiana (B), $M$ schizocarpa (S), hybride $M$ acuminata $\times M$ balbisiana (AB), hybride $M$ acuminata $\times M$ schizocarpa (AS), et par une minuscule les bananiers des sous-espèces de $M$ acuminata : $M$ a banksii (ba), $M$ a burmannica (bu), $M$ a burmannicoides (bs), $M$ errans (e), $M$ a microcarpa (mi), $M$ a malaccensis (ma), $M$ a siamea (si), $M$ a truncata (tr) et $M$ a zebrina $(\mathrm{z})$. Les 2 bananiers diploïdes parthénocarpiques supposés purs balbisiana ont été projetés et sont notés : $B B$ ? $c v$.

\section{DISCUSSION}

\section{Espèces séminifêres}

Les 3 espèces étudiées sont d'effectifs inégaux. Toutefois, $M$ acuminata apparaît la plus variable comme le montraient les études de diversités morphologique 


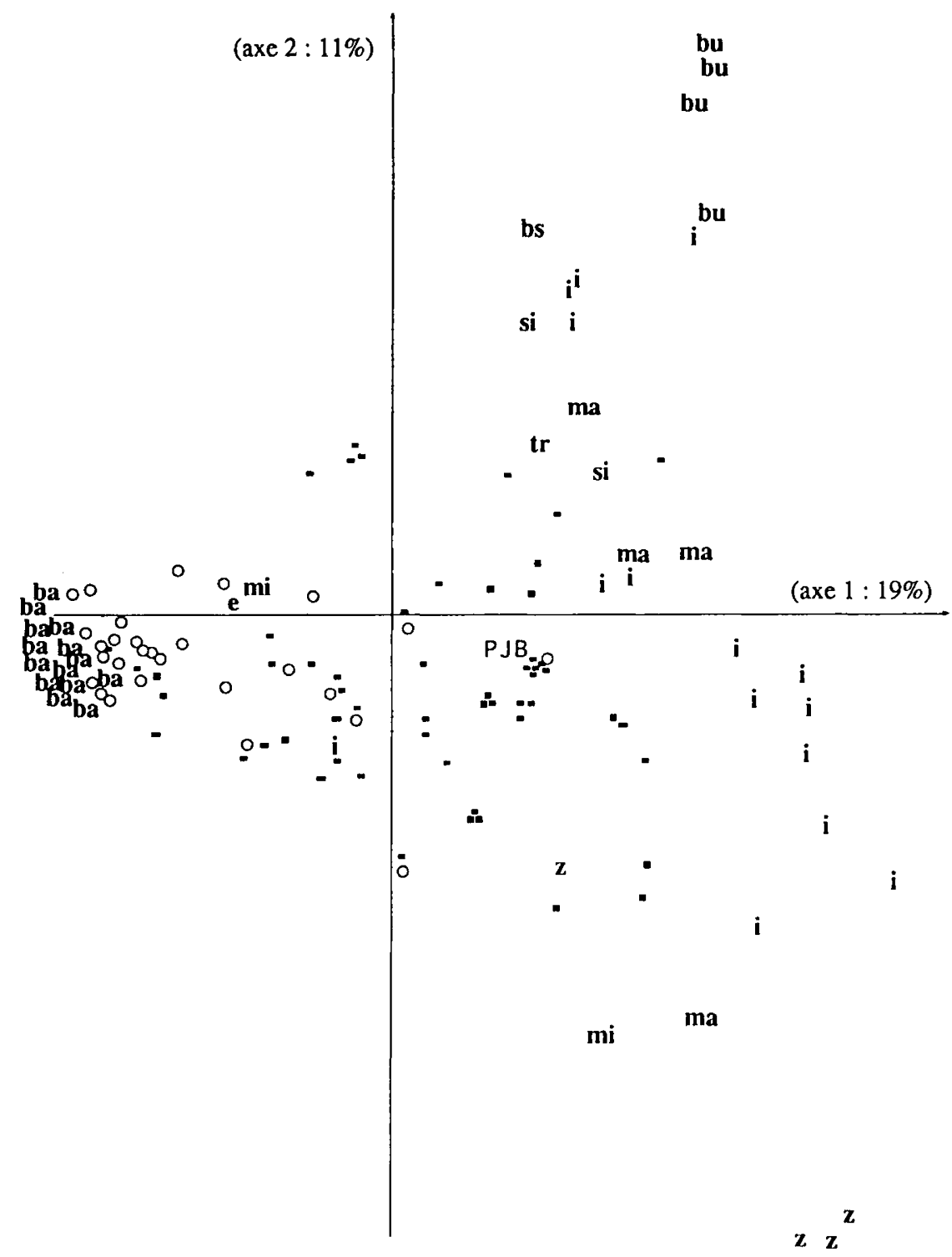

Fig 3. Localisation de 84 bananiers séminifères et parthénocarpiques de $M$ acuminata sur le premier plan de l'AFC qui représente $30 \%$ de la variabilité révélée par 30 sondes nucléaires. Sont représentés par une minuscule les bananiers séminifères : $M a$ banksii (ba), $M$ a burmannica (bu), $M$ a burmannicoides (bs), $M$ errans (e), $M$ a microcarpa (mi), $M$ a malaccensis (ma), $M$ a siamea (si), $M$ a truncata $(\mathrm{tr}), M$ a zebrina $(\mathrm{z})$, les indéterminés (i) et par ( $\square$ ) les bananiers parthénocarpiques, exceptés ceux issus de la prospection en Papouasie Nouvelle-Guinée représentés par (o). PJB indique la localisation du cultivar Pisang Jari Buaya. 
(Simmonds, 1988) et enzymatique (Horry, 1989). Les espèces $M$ acuminata et $M$ balbisiana sont aisément distinguables morphologiquement, et notre analyse reflète clairement cette distinction. Une grande affinité est observée entre les espèces $M$ acuminata et $M$ schizocarpa, confirmant les analyses par électrophorèse d'isozymes de Lebot (1993).

La situation des espèces $M$ acuminata, $M$ acuminata ssp banksii et $M$ schizocarpa est plus ambiguë. Aucune barrière de fertilité n'étant mise en évidence, Simmonds (1962) a intégré banksii comme une sous-espèce de $M$ acuminata, ce que réfute Argent (1976) : la répartition géographique (l'Est de l'aire de répartition de l'espèce) et la biologie florale de banksii en feraient une espèce à part entière. Une analyse des caractères morphologiques en taxonomie numérique (Simmonds et Weatherup, 1988), souligne le caractère particulier de banksii au sein de $M$ acuminata. Notre analyse oppose clairement les banksii aux autres formes de $M$ acuminata; cependant, l'excellente fertilité des hybrides entre banksii et autres acuminata, n'autorise pas, à notre sens, sa scission de l'espèce $M$ acuminata. L'espèce $M$ schizocarpa est, quant à elle, différenciable des formes de $M$ acuminata par sa morphologie (Tezenas du Montcel, 1990), mais son aire de répartition et son type de reproduction (andromonoïque et non monö̈que comme pour les autres bananiers séminifères) sont identiques à ceux de $M$ a banksii. Nos résultats confirment cette proximité des 2 types; toutefois des allèles spécifiques à $M$ schizocarpa ont été identifiés. Mais les analyses en classification réalisées (non détaillées ici) ne nous ont pas permis de conclure quant à l'appartenance de $M$ schizocarpa à l'espèce $M$ acuminata. Outre un niveau d'homozygotie très élevé, comparable à celui de $M a$ banksii, l'individu de $M$ a errans se positionne nettement dans l'analyse factorielle dans le groupe banksii. $M$ a errans serait donc à replacer comme une forme de $M$ a banksii, comme le suggère Shepherd (1990).

Parmi les autres clones séminifêres de $M$ acuminata, la forme Bornéo, classée morphologiquement comme $M$ a microcarpa se positionne entre les accessions de $M a$ banksii et celles des autres sous-espèces. Trois autres sous-espèces apparaissent bien différenciées. $M$ a burmannica est originaire de la zone Nord-Ouest de l'aire de répartition de l'espèce (Inde, Birmanie, nord-Thaïlande) à laquelle nous incluons le clone de $M$ a burmannicoides, la séparation en 2 sous-espèces particulières semblant injustifiée. $M$ a malaccensis représente un compartiment Thaïlande-Malaisie auquel on rattache tout comme Gawel et Jarret (1991a) par analyse du génome chloroplastique, la sous-espèce $M$ a truncata originaire des zones montagneuses de Malaisie. Enfin, nous distinguons la sous-espèce $M$ a zebrina de la zone indonésienne à laquelle pourrait être associée $M$ a microcarpa. Les individus de $M$ a siamea analysés, originaires de Thaïlande, apparaissent comme intermédiaires entre les sous-espèces $M$ a burmannica et $M$ a malaccensis. Le statut de sous-espèce pour ces individus siamea ne se justifie pas aux vues de nos résultats.

La compartimentation due à l'isolement géographique et aux différences structurales chromosomiques n'est pas totale et les flux de gènes ont pu être importants, comme le souligne cette analyse.

\section{Variétés parthénocarpiques}

Longtemps ignorée, Musa schizocarpa pourrait également avoir joué un rôle dans l'évolution de certains cultivars. Nous avons prouvé, au niveau moléculaire, l'exis- 
tence d'hybrides séminifères et surtout d'hybrides parthénocarpiques entre les 2 espèces $M$ schizocarpa et $M$ acuminata. De même origine que $M$ schizocarpa, $M$ a banksii apparaît comme le parent acuminata de ces hybrides AS.

L'existence de cultivars purement balbisiana pouvait remettre en cause l'apparition de la parthénocarpie chez $M$ acuminata. Toutefois notre étude a montré que les 2 cultivars considérés morphologiquement comme pouvant être des diploïdes $\mathrm{BB}$, étaient en fait des hybrides $\mathrm{AB}$ mais différents de ceux que l'on connaissait déjà (notés BB sur la fig 3 ). Comme pour $M$ balbisiana, il n'a pas été trouvé de cultivars purement schizocarpa. Notre étude supporte donc l'hypothèse de l'apparition de la parthénocarpie uniquement chez $M$ acuminata.

La majorité des clones parthénocarpiques issus de la prospection en Papouasie Nouvelle-Guinée sont très proches de $M$ a banksii également originaire de cette zone. $M$ a banksii apparaît comme la principale sous-espèce à l'origine de ces accessions. Hormis quelques exceptions, les cultivars n'appartenant pas au complexe banksii forment un continuum entre les différentes sous-espèces de $M$ acuminata. Leur niveau d'hétérozygotie très élevé suppose une structure génétique hybride, et il est vraisemblable que pour la plupart, ils ont pour origines plusieurs sous-espèces. Une classification de ces cultivars qui serait dérivée de la structuration en sous-espèces est délicate, aucun allèle sub-spécifique n'ayant été mis en évidence au niveau des variétés séminifêres acuminata étudiées. Une analyse plus approfondie des données obtenues s'avère nécessaire. Toutefois, notre analyse permet le regroupement de certains cultivars. Ainsi, 6 accessions parthénocarpiques sont génétiquement proches du clone Pisang Jari Buaya (PJB, fig 3) chez lequel une résistance vis-à-vis des nématodes a été observée (Pinochet et Rowe, 1978). Ces informations sont d'une grande utilité non seulement pour la gestion des collections, mais également pour la sélection, permettant à terme la constitution de groupes d'aptitudes à la combinaison.

Par une analyse génétique notre étude a permis de discuter la classification de $M$ acuminata et de montrer le rôle de $M$ schizocarpa. Peu d'accessions représentent en collection les espèces $M$ balbisiana et $M$ schizocarpa ainsi que les différentes sousespèces de $M$ acuminata; de nouvelles prospections sont indispensables à une étude plus approfondie.

Une hérédité maternelle du génome chloroplastique et une hérédité paternelle du génome mitochondrial ont été trouvées chez le bananier (Fauré et al, 1993c). La poursuite d'analyses RFLP à l'aide de sondes cytoplasmiques et leurs comparaisons avec les autres marqueurs permettront de continuer à rechercher chez les diploïdes, ainsi que chez les triploïdes, l'origine des génomes acuminata dans les différentes sous-espèces.

\section{REMERCIEMENTS}

Nous remercions l'INIBAP et J Daniells du QDPI (Australie), de nous avoir procuré les clones de la prospection en Papouasie Nouvelle-Guinée. Cette étude a été financée par le CIRAD et par le programme STD2 de la CEE (TS 2A-0094-F(SD)). 


\section{RÉFÉRENCES}

Argent GCG (1976) The wild bananas of Papua New-Guinea. Notes Roy Bot Gard Edinb 35, 77-114

Bakry F, Horry J, Teisson C, Tezenas du Montcel H, Ganry J (1990) L'amélioration génétique des bananiers à l'IRFA/CIRAD. Fruits, numéro spécial bananes, 25-40 Cheesman EE (1948) Classification of the bananas. III : critical notes on the species $M$ paradisiaca, $M$ sapientum. Kew Bull 2, 106-117

De Langhe EA, Devreux M (1960) Une sous-espèce nouvelle de Musa acuminata Colla. Bull Jard Bot Brux 30, 355-388

Dessauw D (1987) Étude de la stérilité du bananier (Musa spp) et des relations cytotaxonomiques entre Musa acuminata et Musa balbisiana Colla. Thèse de $3^{\mathbf{e}}$ cycle, Université Paris XI

Dodds KS (1943) Genetical and cytological studies of Musa. V. Certain edible diploids. $J$ Genet 45, 114-137

Fauré S, Bakry F, González de León D (1993a) Cytogenetical studies of some diploid bananas. In: international symposium on genetic improvement of bananas for resistance to diseases and pests, Montpellier, France (à paraitre)

Fauré S, Noyer JL, Horry JP, Bakry F, Lanaud C, González de León D (1993b) A molecular marker-based linkage map of diploid bananas (Musa acuminata). Theor Appl Genet (à paraître)

Fauré S, Noyer JL, Carreel F, Horry JP, Bakry F, Lanaud C (1993c) Maternal inheritance of chloroplast genome and paternal inheritance of mitochondrial genome in bananas (Musa acuminata). Curr Genet (à paraître)

Gawel NJ, Jarret RL (1991a) Chloroplast DNA restriction fragment length polymorphisms (RFLPs) in Musa species. Theor Appl Genet 81, 783-786

Gawel NJ, Jarret RL (1991b) A modified CTAB DNA extraction procedure for Musa and Ipomoea. Plant Mol Biol Rep 9, 262-266

Hoisington D (1992) Laboratory protocols: CIMMYT. Appl molecular genetics laboratory Mexico, DF : CIMMYT

Horry JP (1989) Chimiotaxonomie et organisation génétique dans le genre Musa. Thèse de 3ème cycle, Université Paris XI

Lanaud C, Tezenas du Montcel H, Jolivot MP, Glaszmann JC, González de León D (1992) Variation of ribosomal gene spacer length among wild and cultivated banana. Heredity 68, 147-156

Lebot V, Aradhya KM, Manshardt R, Meilleur B (1993) Genetic relationships among cultivated bananas and plantains from Asia and the Pacific. Euphytica 67, 163-175

Pinochet J, Rowe P (1978) Reaction of two banana cultivars to three different nematodes. Plant Dis Report 62, 727-729

Sharrock SL (1990) Collecting Musa in Papua New Guinea. In: Identification of genetic diversity in the genus Musa (Jarret RL, ed). Los Baños, Philippines, 5-10 septembre 1988, INIBAP, Montpellier France, 140-157

Shepherd K, Ferreira FR (1982) The Papua New Guinea biological foundation's banana collection at Laloki, Port Moresby, PNG. Report submitted to IBPGR, Rome, Italy 
Shepherd K (1990) Observations on Musa taxonomy A note on Musa germplasm of the Philippines. In: Identification of genetic diversity in the genus Musa (Jarret RL, ed). Los Baños Philippines, 5-10 septembre 1988, INIBAP, Montpellier, France, 158-165

Simmonds NW, Shepherd K (1955) The taxonomy and origins of the cultivated bananas. J Linn Soc Lond Bot 55, 302-312

Simmonds NW (1962) The evolution of the bananas. Longmans Tropical Sciences Series, London

Simmonds NW, Weatherup TC (1988) Numerical taxonomy of the wild bananas (Musa). New Phytol 115, 567-571

Tezenas du Montcel H (1990) M acuminata subspecies banksii: status and diversity. In : Identification of genetic diversity in the genus Musa (Jarret RL, ed). Los Baños Philippines, 5-10 septembre 1988, INIBAP, Montpellier, France, 211-218 